\title{
LPF Y AUTOPERCEPCIÓN
}

\section{CLP AND SELF-PERCEPTION}

\author{
C. Tacuri Ayavaca ${ }^{1}, \mathrm{~S}$ Reinoso Quezada ${ }^{2}$, \\ ${ }^{1}$ Egresada de la Facultad de Odontología, Universidad Católica de Cuenca, Cuenca, Ecuador. \\ https://orcid.org/0000-0002-8945-6391 \\ ${ }^{2}$ Cirugía Maxilo y Craneo-Facial, Hospital Vicente Corral Moscoso, Hospital del Río Cuenca, Ecuador; \\ Docencia de Cirugía en Universidad Católica de Cuenca, Ecuador \\ *clautacuria10@ hotmail.com \\ DOI: https://doi.org/10.31984/oactiva.v6i3.613
}

\section{Resumen}

Objetivos: conocer la autopercepción de los niños con Labio y Paladar Fisurado de la Clínica Multidisciplinaria de la Universidad Católica de Cuenca. Materiales y métodos: La presente investigación es un estudio descriptivo- transversal. La muestra consistió en 78 pacientes que fueron atendidos en la Clínica Multidisciplinaria de Labio y Paladar Fisurado de la Universidad Católica de Cuenca de ambos sexos. Para la toma de datos se utilizó una encuesta, contando con consentimiento y asentimiento informados. Información que será organizada, analizada y tabulada en una base de datos en Excel Microsoft Office. Resultados: Respecto a la autopercepción de los pacientes con labio y paladar fisurado un $71.8 \%$ afirma sentirse conforme con su imagen, sin embargo, a un $80.8 \%$ le gustaría cambiar su apariencia de ser posible. En general se recibieron respuestas positivas en este estudio realizado. Conclusiones: Un abordaje multidisciplinario para los pacientes con labio y paladar fisurado, ayuda a mejorar la calidad de vida y mejora la autopercepción de cada uno de ellos.

Palabras clave: labio leporino, fisura del paladar, autopercepción.

\begin{abstract}
Aim: To know the self-perception of children with cleft lip and palate of the Multidisciplinary Clinic of the Universidad Católica de Cuenca. Material and methods: The present research is a descriptive-cross-sectional study. The sample consisted of 78 patients of both sexes who were attended at the Clinica Multidisciplinaria de Labio y Paladar Fisurado de la Universidad Católica de Cuenca. A survey was used for data collection, with informed consent and assent. The information will be organized, analyzed and tabulated in an Excel Microsoft Office database. Results: Regarding the self-perception of patients with cleft lip and palate, $71.8 \%$ stated that they were satisfied with their image; however, $80.8 \%$ would like to change their appearance if possible. In general, positive responses were received in this study. Conclusions: A multidisciplinary approach for cleft lip and palate patients helps to improve the quality of life and improves the self-perception of each one of them.
\end{abstract}

Key words: Cleft lip, cleft palate, self- perception.

\section{Introducción}

El labio y paladar fisurado (LPF), es una de las anomalías congénitas que afectan el área orofacial, causado por un defecto en la embriogénesis que impide la fusión de ciertos procesos faciales durante la primera etapa del embarazo, aunque se desconoce las causas de las mismas, se ha demostrado la existencia de factores genéticos y ambientales que contribuyen el desarrollo de defectos orofaciales, así como la deficiencia de ácido fólico, la edad materna y la acción de agentes teratógenos como el consumo de tabaco, alcohol, plaguicidas, entre otros ${ }^{1-} .4$

Se denomina labio fisurado a aquellas hendiduras en el labio superior, éstas pudiendo ser unilaterales o bilaterales, su tamaño puede variar y ser desde bastante pequeñas y presentarse como pequeñas escotaduras, un labio fisurado completo se presenta cuando la hendidura atraviesa el labio, la línea de la encía superior, el paladar y la base de la nariz. Por el contrario, un paladar fisurado se presenta con una abertura en el techo de la boca, está pudiendo ser aislada o ligada a un labio leporino ${ }^{3,5,6}$

Según el Estudio Colaborativo Latinoamericano de Malformaciones Congénitas (ECLAMC), el LPF se presenta con una prevalencia de 15 por 10000 recién nacidos, siendo el sexo masculino el más afectado por esta patología, predominando el labio leporino unilateral y con mayor frecuencia en el lado izquierdo de la cara ${ }^{4},{ }^{7}$ A nivel mundial se reporta que 
aproximadamente cada 3 minutos, nace un niño con un algún tipo de problema orofacial, si no se brinda un tratamiento adecuado las personas con LPF pueden experimentar eventos letales como muerte prematura, y consecuencias definitivas como problemas al alimentarse, habla y relaciones psicosociales, es por esto que se recomienda que las primeras intervenciones quirúrgicas empiecen en las primeras semanas de vida. ${ }^{8,9}$

\section{Clasificación de labio y paladar fisurado}

Las hendiduras orofaciales se pueden clasificar en labio fisurado con o sin paladar hendido, esto según su fenotipo, las mismas pueden ser:

- Completa

- Incompleta

- Unilateral

- Bilateral ${ }^{8,10}$

Como consecuencia de esta patología encontramos consecuencias estéticas y funcionales como; la deglución, masticación, fonación y respiración. ${ }^{11}$ Las personas que presentan LPF durante su etapa de desarrollo presentan problemas psicosociales los mismos que con el tiempo pueden desarrollar baja autoestima, ansiedad, depresión e inhibición social y timidez, los mismos que se asocian a la visibilidad de las cicatrices. ${ }^{2,7}$

Debido a los mitos y creencias culturales y religiosas, uno de los desafíos que enfrentan los pacientes con LPF son las burlas, el acoso y las miradas por largos periodos de tiempo que reciben a diario. ${ }^{12,13}$ Tener una apariencia diferente y sonar de manera distinta al hablar puede producir graves problemas al momento de comunicarse y relacionarse socialmente, provocando de esta manera problemas de interacción, debido a que los pacientes con LFP buscan ser como todos los demás, sin embargo, estos aspectos negativos se pueden mejorar mediante el apoyo de su entorno familiar y personal profesional. ${ }^{14,15}$

Para garantizar la viabilidad de este estudio se han realizado coordinaciones, con las autoridades institucionales de la Universidad Católica de Cuenca, con la dirección de la carrera de Odontología, la cátedra de investigación de la misma, y la Clínica Multidisciplinaria de Labio y Paladar Fisurado.

\section{Materiales y métodos}

Se realizó un estudio descriptivo- transversal, mediante una encuesta en línea. La población de referencia fueron 78 pacientes entre hombres y mujeres atendidos en la Clínica Multidisciplinaria de Labio y Paladar Fisurado, de la Universidad Católica de Cuenca. Para conocer la autopercepción de los pacientes con labio y paladar fisurado se realizó una encuesta, misma que hace referencia a la autopercepción en cuanto a apariencia y aceptación de cada paciente, encuesta que fue validada por los especialistas del departamento de investigación de la carrera de odontología de la Universidad Católica de Cuenca.
Se incluyeron en el presente estudio a aquellos pacientes que hayan sido tratados en la clínica multidisciplinaria de Labio y paladar fisurado y que hayan aceptado el consentimiento y asentimiento. $\mathrm{Y}$ se excluyeron a pacientes que no hayan contestado la encuesta o se negaron a participar en este estudio, y aquellas encuestas que no tengan todos los campos completados.

Las variables estudiadas comprendieron: sexo (masculino y femenino), edad, gestión escolar, etnia, lugar de residencia y autopercepción.

La información fue organizada, analizada y tabulada en una base de datos en Excel Microsoft Office, utilizando estadística descriptiva para reportar los resultados a través de frecuencias y porcentajes.

\section{Resultados}

Aspectos sociodemográficos

Tabla 1. Aspectos sociodemográficos de los pacientes atendidos en la Clínica Multidisciplinaria de Labio y Paladar Fisurado de la Universidad Católica de Cuenca $(\mathrm{N}=78)$

\begin{tabular}{|c|c|c|c|}
\hline \multicolumn{2}{|c|}{$\begin{array}{l}\text { Variable } \\
\text { sociodemográfica }\end{array}$} & $\mathbf{n}$ & $\%$ \\
\hline \multirow[t]{2}{*}{ Sexo } & Femenino & 37 & $47.4 \%$ \\
\hline & Masculino & 41 & $52.6 \%$ \\
\hline \multirow[t]{3}{*}{ Edad } & $1-9$ & 33 & $42.3 \%$ \\
\hline & $10-18$ & 39 & $50 \%$ \\
\hline & $19-27$ & 6 & $7.6 \%$ \\
\hline \multirow{4}{*}{$\begin{array}{l}\text { Gestión } \\
\text { escolar }\end{array}$} & Sin & 14 & $17.9 \%$ \\
\hline & estudios & 36 & $46.2 \%$ \\
\hline & Primaria & 28 & $35.9 \%$ \\
\hline & Secundaria & & \\
\hline \multirow[t]{5}{*}{ Etnia } & Mestizo & 72 & $92.3 \%$ \\
\hline & Afroecuatori & 0 & $0 \%$ \\
\hline & ana & 2 & $2.6 \%$ \\
\hline & Indígena & 4 & $5.1 \%$ \\
\hline & Blanco & & \\
\hline \multirow{3}{*}{$\begin{array}{l}\text { Lugar de } \\
\text { residencia }\end{array}$} & Cuenca & 42 & $53.8 \%$ \\
\hline & Fuera de & 36 & $46.2 \%$ \\
\hline & Cuenca & & \\
\hline
\end{tabular}

La tabla 1 muestra los resultados de acuerdo con las variables sociodemográficas, en cuanto al sexo hubo mayor prevalencia de pacientes de sexo masculino con un $52.6 \%$ $(n=41)$. El rango de edad con mayor prevalencia en este estudio es el de 10-18 años con un 50\% (n=39). En cuanto a la gestión escolar, la mayoría de pacientes se encuentra 
cursando la primaria con un porcentaje del $46.2 \%(n=32)$. El $92.3 \%(\mathrm{n}=72)$ de los pacientes encuestados se identifica con la etnia mestiza y el $53.8 \%(n=42)$ de pacientes residen en la ciudad de Cuenca. fisurado

Autopercepción de pacientes con labio y paladar

Tabla 2. Autopercepción de los pacientes con labio y paladar fisurado de la Clínica Multidisciplinaria de Labio y Paladar Fisurado de la Universidad Católica de Cuenca en porcentaje $(\mathrm{N}=78)$

\begin{tabular}{|c|c|c|}
\hline $\begin{array}{l}\text { Pregunta } \\
\text { a) ¿Te sientes conforme } \\
\text { con tu imagen? }\end{array}$ & $\begin{array}{c}\text { SI } \\
71.8 \%\end{array}$ & $\begin{array}{c}\text { NO } \\
28.2 \%\end{array}$ \\
\hline $\begin{array}{l}\text { b) ¿Te gustaría cambiar } \\
\text { algún aspecto de tu imagen } \\
\text { si así lo pudieras? }\end{array}$ & $80.8 \%$ & $19.2 \%$ \\
\hline $\begin{array}{l}\text { c) ¿Tienes problemas para } \\
\text { comunicarte en tu vida } \\
\text { cotidiana? }\end{array}$ & $47.4 \%$ & $52.6 \%$ \\
\hline $\begin{array}{l}\text { d) ¿Te molesta sonreír } \\
\text { delante de otras personas? }\end{array}$ & $38.5 \%$ & $61.5 \%$ \\
\hline $\begin{array}{l}\text { e) ¿Te molesta hablar } \\
\text { delante los demás? }\end{array}$ & $33.3 \%$ & $66.7 \%$ \\
\hline $\begin{array}{l}\text { f) ¿Te preocupa lo que los } \\
\text { demás piensen de ti? }\end{array}$ & $26.9 \%$ & $73.1 \%$ \\
\hline $\begin{array}{l}\text { g) ¿Te resulta } \\
\text { relacionarte con otras } \\
\text { personas? }\end{array}$ & $50 \%$ & $50 \%$ \\
\hline $\begin{array}{l}\text { h) ¿Tienes una actitud } \\
\text { positiva contigo mismo/a? }\end{array}$ & $87.2 \%$ & $12.8 \%$ \\
\hline $\begin{array}{l}\text { i) ¿Sientes respeto por ti } \\
\text { mismo? }\end{array}$ & $96.2 \%$ & $3.8 \%$ \\
\hline $\begin{array}{l}\text { j) ¿Eres popular entre los } \\
\text { chicos de tu edad? }\end{array}$ & $28.3 \%$ & $71.8 \%$ \\
\hline $\begin{array}{l}\text { k) ¿Te preocupa la opinión } \\
\text { que tienen los demás sobre } \\
\text { tu imagen? }\end{array}$ & $33.3 \%$ & $66.7 \%$ \\
\hline $\begin{array}{l}\text { I) ¿Alguna vez has } \\
\text { escuchado burlas hacia ti? }\end{array}$ & $56.4 \%$ & $43.6 \%$ \\
\hline $\begin{array}{l}\text { m) ¿Te cuesta concentrarte } \\
\text { en los estudios u otras } \\
\text { actividades? }\end{array}$ & $43.6 \%$ & $56.4 \%$ \\
\hline $\begin{array}{l}\text { n) ¿Sientes que tu entorno } \\
\text { puede confiar en ti? }\end{array}$ & $93.6 \%$ & $6.4 \%$ \\
\hline $\begin{array}{l}\text { o) ¿Te sientes siempre } \\
\text { cómodo en la escuela? }\end{array}$ & $67.9 \%$ & $32.1 \%$ \\
\hline $\begin{array}{l}\text { p) ¿Sientes que tus padres } \\
\text { son muy protectores } \\
\text { contigo? }\end{array}$ & $74.4 \%$ & $25.6 \%$ \\
\hline
\end{tabular}

Más de la mitad de los pacientes, un $71.8 \%$ se siente conforme con su imagen, sin embargo, al $80.8 \%$ de pacientes encuestados les gustaría cambiar su aspecto si lo pudiera. Dentro del ámbito social, un $52.6 \%$ no tiene problemas a la hora de comunicarse diariamente. Además, la mayoría de los encuestados manifestó que un $61.5 \%$ sentían cierta molestia al sonreír y un $66.7 \%$ de pacientes sentían molestias al hablar frente a otras personas, un $73.1 \%$ de pacientes manifestó que les producía cierta preocupación lo que los demás piensen de ellos. Con respecto a las relaciones personales, existió un equilibrio $(50 \%-50 \%)$ en cuanto a la dificultad de relacionarse con otras personas.

En su mayoría, el $87.2 \%$ de pacientes tienen una actitud positiva y un $96.2 \%$ de encuestados sienten respeto por sí mismos, así también, a pesar de que el $71.8 \%$ de pacientes manifestó no ser populares entre los chicos de su edad, en su gran mayoría, un $66.7 \%$ manifiesta no tener preocupación sobre la opinión de los demás sobre su imagen, a pesar de que un $56.4 \%$ de pacientes muchas veces han escuchado burlas sobre su apariencia.

En general, un $56.4 \%$ de pacientes manifiesta que la autopercepción percibida no ha provocado desconcentración en estudios u otras actividades diarias, un $93.6 \%$ de pacientes se considera a sí mismos como personas confiables y manifiestan en su mayoría un $67.9 \%$ estar cómodos en ambientes educativos y laborales. Finalmente, un $74.4 \%$ de pacientes encuestados manifiestan que sus padres son o han sido muy sobreprotectores debido a esta condición.

\section{Discusión}

El objetivo de la presente investigación fue conocer la autopercepción de los niños con Labio y Paladar Fisurado de la Clínica Multidisciplinaria de la Universidad Católica de Cuenca. En un estudio realizado por González y cols. manifestaron que al igual que en nuestro estudio, los pacientes con LPF no sienten molestia alguna por su apariencia, a pesar de que una gran cantidad de ellos afirmó haber sufrido burlas en su vida cotidiana, sin embargo, su desarrollo social no se ha visto limitado por esta acción 7 . Vinaccia y cols. afirma que la autoimagen de los pacientes con labio y paladar fisurado es mucho más vulnerable esto debido a los defectos visibles en la facies, sin embargo, afirma que la autopercepción no se ha visto afectada por estos defectos congénitos, resultados coincidentes con el presente estudio. Además, reporta que esto no influye en las actividades diarias, y tampoco afecta las habilidades sociales y actividades de la vida cotidiana de cada uno de los pacientes. ${ }^{16}$

Así como en la presente investigación se demuestra la sobreprotección de los padres con los pacientes con labio y paladar fisurado, Márquez afirma que el nacimiento de un niño con LPF provoca en los padres un instinto de sobreprotección, teniendo un vínculo afectivo mucho mayor que en niños sin este tipo de malformación. Sin embargo, existen casos reportados, que padres manifiestan cierto rechazo fren- 
te a estas situaciones, lo que provoca una difícil adaptación y la estructura familiar muchas veces se encuentra afectada. ${ }^{17}$

En un estudio realizado por Brent $\mathrm{R}$ y cols, mencionan que los pacientes con labio y paladar fisurado desarrollan autopercepciones negativas, incluyendo la apariencia, comunicación e interacción con terceros, sin embargo, todo nuestro grupo encuestado manifiestan tener una buena autopercepción. ${ }^{18}$

\section{Conclusiones}

Luego del estudio y análisis realizado, consideramos que un manejo multidisciplinario para los pacientes con labio y paladar fisurado es el punto clave en el tratamiento, ya que el mismo se enfoca en aspectos estéticos y funcionales, teniendo en cuenta que uno de los puntos más fundamentales en el desarrollo cognitivo es el aspecto psicológico, razón por la cual el manejo psicológico para el niño y la familia son un pilar fundamental en este proceso. Todo esto, con la finalidad de ayudar a la autopercepción de cada uno de los pacientes, brindándoles una mejor calidad de vida, evitando sentir molestias por si mismos a la hora de hablar, sonreír y realizar actividades. A pesar de lo expuesto, en este trabajo de investigación hemos alcanzado una respuesta positiva en cuanto a autoaceptación.

Conflicto de intereses: Los autores declaran no tener conflicto de intereses.

\section{Referencias}

1 Guerrero-Abello P, Ariza-Araujo Y, Caycedo-García DJ. Necesidad de guías clínicas para el manejo integral de pacientes con labio paladar hendido. Rev SALUD PÚBLICA. 2016;13.

2 Al-Namankany A, Alhubaishi A. Effects of cleft lip and palate on children's psychological health: A systematic review. J Taibah Univ Med Sci. 5 de junio de 2018;13(4):311-8.

3 Fritzsche S. Care of the Asian American Child With Cleft Lip or Palate. Plast Surg Nurs Off J Am Soc Plast Reconstr Surg Nurses. diciembre de 2019;39(4):142-7.

4 Quezada SJR, Carrasco AMT, Caparó EV. Determinantes sociales adversos y Riesgo de labio y paladar fisurados Estudio de casos y controles. Rev Fac Cienc Médicas Univ Cuenca. 31 de agosto de 2020;38(2):ículo 4:1-8.

5 Pons-Bonals A, Pons-Bonals L, Hidalgo-Martínez SM, Sosa-Ferreyra CF. Estudio clínico-epidemiológico en niños con labio paladar hendido en un hospital de segundo nivel. Bol Méd Hosp Infant Méx. 2017;107-21.

6 Crockett DJ, Goudy SL. Cleft lip and palate. Facial Plast Surg Clin N Am. noviembre de 2014;22(4):573-86.

7 González-Carrera MC, Téllez-Merchán M, Canchano F, Rojas Y, Trujillo MI. Calidad de vida y salud oral en una población colombiana con labio y/o paladar fisurado. Univ Odontológica. 2011;30(64):73.

8 Sandy J, Davies A, Humphries K, Ireland T, Wren Y. Cleft lip and palate: Care configuration, national registration, and research strategies. J World Fed Orthod. octubre de 2020;9(3):S40-4.

9 Yates D, Allareddy V, Caplin J, Yadav S, Markiewicz MR. An Overview of Timeline of Interventions in the Continuum of Cleft Lip and Palate Care. Oral Maxillofac Surg Clin N Am. mayo de 2020;32(2):177-86.

10 Taib BG, Taib AG, Swift AC, van Eeden S. Cleft lip and palate: diagnosis and management. Br J Hosp Med Lond Engl 2005. octubre de 2015;76(10):584-5, 588-91.

11 Losier V, Cyr C, Dubois-Comtois K. International adoption of cleft lip and palate children: Attachment, behavior problems, and parental sensitivity. J Appl Dev Psychol. 1 de mayo de 2020;68:101138.

12 Abualfaraj R, Daly B, McDonald F, Scambler S. Cleft lip and palate in context: Learning from, and adding to, the sociological literature on long-term conditions. Health ( $\mathrm{N}$ Y). 1 de julio de 2018;22(4):372-88.

13 Shaye D, Liu CC, Tollefson TT. Cleft Lip and Palate: An Evidence-Based Review. Facial Plast Surg Clin N Am. agosto de 2015;23(3):357-72.

14 Bickham RS, Ranganathan K, Wombacher NR, Shapiro DN, Carlozzi NE, Baker MK, et al. Speech Perceptions and Health-Related Quality of Life Among Children With Cleft Lip and Palate. J Craniofac Surg. julio de 2017;28(5):12648.

15 Ardouin K, Hare J, Stock NM. Emotional Well-Being in Adults Born With Cleft Lip and/or Palate: A Whole of Life Survey in the United Kingdom. Cleft Palate-Craniofacial J Off Publ Am Cleft Palate-Craniofacial Assoc. julio de 2020;57(7):877-85.

16 Vinaccia S, Quiceno JM, Fernández H, Calle LA, Naranjo M, Osorio J, et al. Autoesquemas y habilidades sociales en adolescentes con diagnóstico de labio y paladar hendido. Pensam Psicológico. 2008;4(10):123-35.

17 Márquez, M. La familia del niño(a) con Hendidura Labio Palatina. Acta Odontológica Venez [Internet]. 2013 [citado 15 de marzo de 2021];51. Disponible en: https://www.actaodontologica.com/ediciones/2013/1/art23/

18 Collett BR, Speltz ML. Social-Emotional Development of Infants and Young Children With Orofacial Clefts: Infants Young Child. octubre de 2006;19(4):262-91.

Recibido: 31 de marzo de 2021

Aceptado: 29 de abril de 2021 